Relations industrielles

Industrial Relations

\title{
Morin, Fernand et Jean-Yves Brière, Le droit de l'emploi au Québec
}

\section{Gilles Trudeau}

Volume 55, numéro 2, 2000

URI : https://id.erudit.org/iderudit/051313ar

DOI : https://doi.org/10.7202/051313ar

Aller au sommaire du numéro

Éditeur(s)

Département des relations industrielles de l'Université Laval

ISSN

0034-379X (imprimé)

1703-8138 (numérique)

Découvrir la revue

Citer ce compte rendu

Trudeau, G. (2000). Compte rendu de [Morin, Fernand et Jean-Yves Brière, $L e$ droit de l'emploi au Québec]. Relations industrielles / Industrial Relations, 55(2), 353-356. https://doi.org/10.7202/051313ar

Tous droits réservés (C Département des relations industrielles de l'Université Laval, 2000
Ce document est protégé par la loi sur le droit d'auteur. L’utilisation des services d'Érudit (y compris la reproduction) est assujettie à sa politique d'utilisation que vous pouvez consulter en ligne.

https://apropos.erudit.org/fr/usagers/politique-dutilisation/ 


\section{Recensions}

\section{Book Reviews}

\section{Le droit de l'emploi au Québec}

par Fernand MORIN et Jean-Yves BRIÈRE, Montréal : Wilson et Lafleur, 1998, 1448 p., ISBN 2-89127-423-7.

Par leur magnifique ouvrage publié il y a déjà plus d'un an, les auteurs ont relevé avec succès un défi de taille : présenter de façon complète et accessible l'ensemble des règles formant le droit du travail en vigueur au Québec. Leur travail colossal fait œuvre de pionnier à plusieurs égards.

L'originalité de l'ouvrage ressort tout d'abord de son titre même : le droit de l'emploi au Québec. Cette désignation du droit applicable au travail salarié a été préférée à celle plus traditionnelle de « droit du travail ». Les auteurs avancent plusieurs raisons justifiant leur orientation. Ils veulent par celle-ci souligner que leur livre aborde aussi bien le sujet de la relation d'emploi, la personne employée, que l'objet de cette relation, soit la prestation de travail. Cela les conduit à analyser non seulement les règles formant le cour même du droit du travail traditionnel, comme celles applicables au contrat individuel de travail ou à la négociation collective, mais aussi «plusieurs questions limitrophes, connexes ou complémentaires au travail, en tenant compte de la personne qui est le sujet-objet de l'opération et qui lui donne sa spécificité : libertés publiques, formation professionnelle, carrière, accident du travail, régime de rentes, avantages sociaux, etc. Il s'agit, d'une certaine manière, d'embrasser les aspects juridiques de l'ensemble des péripéties susceptibles de survenir au cours de la vie professionnelle d'un salarié. » Les auteurs veulent aussi marquer l'im- portance donnée dans leur analyse à l'évolution contemporaine de la forme juridique de l'emploi. Celui-ci n'est plus aussi monolithique qu'à l'époque pas si lointaine du fordisme et de son corollaire, le travail salarié. Aujourd'hui, l'emploi recouvre davantage qu'autrefois des réalités multiples comme le travail à la pige, le travail via des agences de location, le travail autonome, plus ou moins dépendant, la sous-traitance, etc. Les auteurs soulignent enfin avec justesse le caractère évolutif de la dénomination de la branche du droit qu'ils étudient. Cette évolution est conditionnée par celle de la réalité économique et sociale à laquelle elle s'applique : qualifiée de «législation industrielle » au début de l'industrialisation, cette branche du droit objectif fut tour à tour désignée comme le «droit ouvrier» puis, à partir du début des années soixante, comme le « droit du travail ». Aujourd'hui, on doit convenir avec les auteurs que l'appellation « droit de l'emploi » chapeaute mieux cette réalité juridique plus large et multiforme que jamais.

L'analyse approfondie d'un objet aussi large posait nécessairement des difficultés de structure dans la conception de l'ouvrage. À cet égard aussi, les auteurs ont fait preuve d'une originalité et d'une audace qui ont bien servi l'intelligence de leur analyse. Ils ont utilisé une division en six titres, chacun composé de quelques chapitres. Le premier titre décrit la notion même de droit de 
l'emploi, ses sources et ses caractéristiques générales. À noter particulièrement le chapitre trois de ce titre qui présente l'évolution de l'intervention de l'État dans le domaine du travail. Même si elle demeure très succincte, cette analyse permet de bien comprendre les différentes raisons qui ont motivé l'État à intervenir dans ce secteur, les objectifs poursuivis dans ces interventions et les formes qu'elles ont revêtues. Il s'agit là d'un préalable nécessaire à la bonne compréhension du droit de l'emploi actuel.

Les titres deux et trois constituent la description et l'analyse de ce qui est ailleurs appelé le droit des rapports individuels du travail ou encore des aspects juridiques de la gestion des ressources humaines. Les auteurs, plutôt que de suivre cette classification imprécise et quelque peu dépassée, ont préféré étudier de façon séparée les règles applicables au contrat de travail telles qu'elles découlent du Code civil du Québec (titre II) et celles insérées dans le lien d'emploi ou autour de celui-ci par les différentes lois du travail d'application générale (titre III). Cette structure favorise la clarté et l'homogénéité dans le développement de l'analyse. Ainsi, le titre II permet une présentation complète des différentes étapes du lien d'emploi dans la logique contractuelle du droit commun. Le titre trois consacre un chapitre à chacune des grande lois québécoises s'intéressant d'une façon ou d'une autre au travail : la Charte des droits et libertés de la personne et la Charte de la langue française (chapitre III-1), la Loi sur les normes du travail (chapitre III-2), la Loi sur les accidents du travail et les maladies professionnelles (chapitre III-3), la Loi sur la santé et la sécurité du travail (chapitre III-4), la Loi sur les décrets de convention collective (chapitre III-5) et la Loi sur les relations du travail, la formation professionnelle et la gestion de la main-d'auvre dans l'industrie de la construction (chapitre III-6). Un dernier chapitre est consacré à six autres lois ou domaines particuliers de l'emploi, notamment l'équité salariale, la protection des renseignements personnels, les régimes de retraite et la formation professionnelle (chapitre III-7). Pour chacune des lois étudiées dans le titre III, les auteurs abordent sa raison d'être, ses origines et ses objectifs, son champ d'application, son contenu général et enfin, son fonctionnement et son administration.

Cette répartition de la matière entre les titres II et III empêche le lecteur d'avoir accès en un seul lieu de l'ouvrage, à une analyse complète des règles tant substantives que procédurales applicables à une étape précise du lien d'emploi. Par exemple, pour connaître toutes les règles applicables à la formation du lien d'emploi, le lecteur devra notamment consulter à la fois le chapitre II-1 (L'engagement) et le chapitre III-1 (Les chartes et le droit de l'emploi). Les auteurs ont toutefois contourné cet inconvénient de façon satisfaisante en relevant systématiquement dans le titre II en quoi le droit commun et la liberté contractuelle qu'il établit sont modifiés par les règles substantives et généralement d'ordre public provenant des diverses lois abordées dans le titre III. De plus, un système efficace de renvois permet de faire facilement le pont entre la règle substantive exposée dans le titre II et l'analyse plus complète de la loi d'où elle provient dans le titre III.

Par ailleurs, la description des objets traités dans les titres II et III permet de constater à quel point l'analyse des auteurs est large et diverse. Par exemple, ils ont couvert des sujets aussi variés mais complémentaires que l'étude détaillée des règles énoncées dans le Code civil du Québec quant à la formation valide d'un contrat individuel de travail que la présentation générale du régime particulier de travail applicable aux artistes québécois. Le travail des auteurs à cet égard est particulièrement remarquable.

Le titre IV porte exclusivement sur le droit des rapports collectifs du travail. 
Cette analyse, plus traditionnelle, débute par les règles applicables spécifiquement à l'acteur syndical : la reconnaissance de la liberté syndicale et sa protection de même que les aspects juridiques du gouvernement syndical (chapitre IV-1). Suivent les chapitres qui collent à la chronologie du régime de la négociation collective à la manière du Code du travail : accréditation (chapitre IV-2), négociation collective (chapitre IV-3) et convention collective (chapitre IV-4). Un chapitre décrivant le régime particulier des services et du secteur public clôt le titre IV.

Les auteurs ont décidé de regrouper l'analyse de tous les recours administratifs et judiciaires existant dans le droit de l'emploi au sein du titre V intitulé Les contentieux de l'emploi. À l'instar de la division établie entre le droit substantif applicable à la relation individuelle d'emploi (titre II) et la structure des différentes lois du travail (titre III), ce choix est posé au détriment d'une analyse juridique intégrée et complète de chaque étape du lien d'emploi. Par contre, il favorise la clarté et l'efficacité dans le traitement puisque des recours semblables existent dans plusieurs lois du travail différentes. Le même système de renvois efficace est utilisé pour faire les liens nécessaires avec le contenu des titres II, III et IV. Le chapitre V-1 analyse les recours qui permettent le contrôle de certaines décisions de l'employeur. On y voit plus particulièrement le recours disponible pour contester les mesures de représailles qu'un employeur pourrait imposer pour avoir exercé un droit établi dans le Code du travail, dans la Loi sur les normes, ou dans plusieurs autres lois créant des droits à l'égard des salariés. La dernière section de ce chapitre est consacrée à la description de l'important recours institué par les articles 124 et suiv. de la Loi sur les normes du travail concernant le congédiement injustifié. Le deuxième chapitre du titre $V$ est particulièrement important puisqu'il regroupe tous les recours qui relèvent de la compétence des tribunaux de droit commun. On y retrouve les réclamations qui découlent du contrat de travail, qu'elles concernent l'exécution forcée des obligations de celui-ci ou un équivalent compensatoire. De plus, le chapitre contient une analyse non seulement des réclamations civiles formulées à partir des lois du travail d'application générale comme la Loi sur les normes du travail, la Loi sur les décrets de convention collective, mais celles pouvant être poursuivies à l'égard des administrateurs de l'employeur et celles appartenant aux créanciers de l'employé. Le même chapitre aborde aussi la question particulière de la responsabilité civile du syndicat du fait de la grève. Il traite enfin succinctement de quatre recours extraordinaires de la compétence des tribunaux de droit commun et qui ont une incidence significative sur le droit de l'emploi : la révision judiciaire des décisions des tribunaux spécialisés, l'injonction, la requête en jugement déclaratoire et l'outrage au tribunal. La complexité des matières abordées dans certaines lois du travail a amené le législateur à mettre sur pied des procédures d'enquête et d'adjudication qui relèvent davantage de l'Administration. Le chapitre V-3 en analyse les plus importantes dont celle applicable à la protection des renseignements personnels, celle garantissant une représentation syndicale juste et loyale, celle rattachée à la Charte des droits et libertés de la personne et celle prévalant en matière de santé et de sécurité au travail. Enfin le chapitre se termine sur l'analyse du contentieux pénal, un contentieux qui prévaut encore en certains domaines du droit de l'emploi.

Le dernier titre, le titre VI, joue le rôle de conclusion et d'épilogue, en abordant la délicate question des emplois atypiques et précaires. Les auteurs y démontrent que le droit du travail traditionnel, quoique encore applicable à maints égards à ces catégories hétéroclites d'emplois, y est beaucoup plus à 
l'étroit. Dans cette perspective, l'efficacité de son rôle protecteur peut certainement être remis en question comme le souligne fort justement le propos suivant : «En somme, on ne peut cultiver l'insécurité et la précarisation d'une part et, d'autre part exiger la loyauté, le dévouement, la disponibilité et l'esprit d'initiative. Pour ces raisons, nous devons constater l'inadaptation des lois actuelles aux situations présentes et pressantes ».

L'analyse des auteurs est aussi originale dans son contenu et son développement. Il s'agit avant tout d'un livre de droit objectif. Il décrit, organise et analyse les différentes règles juridiques applicables à l'emploi. Les auteurs le font fort bien, permettant au lecteur, qu'il soit étudiant, professionnel ou chercheur, d'avoir un bon aperçu des multiples règles de droit en vigueur en ce domaine. Les auteurs ont su demeurer à un niveau de généralité suffisamment élevé pour assurer un accès facile à leur analyse. Par contre, elle revêt un caractère assez approfondi et détaillé pour être aussi immédiatement utile dans une perspective professionnelle. De plus, il s'agit d'un ouvrage à la fois analytique et synthétique, qui évite de simplement paraphraser les dispositions légales ou de citer de longs extraits jurisprudentiels. Au-delà de son caractère juridique, l'ouvrage insiste beaucoup sur les aspects institutionnels de l'emploi. En fait, bien des dispositions du droit de l'emploi ne pourraient pas être bien comprises sans leur insertion dans leur contexte historique et institutionnel. L'analyse des auteurs est exemplaire à cet égard. Il s'agit tout aussi bien d'un ouvrage de relations industrielles que de droit. Par ailleurs, l'ouvrage n'est pas purement théorique. Il ne se limite pas simplement à la description et à l'analyse du contenu de la règle de droit. Il en décrit aussi la mise en œuvre, en relève les difficultés d'application et même les limites de son efficacité. Il faut souligner ici l'apport particulier du titre $\mathrm{V}$ dans lequel on décrit de façon très concrète le fonctionnement des différents recours reliés au droit de l'emploi. Les auteurs y mettent à contribution non seulement leur formation académique mais aussi leur expérience dans la pratique professionnelle.

La qualité de la rédaction de l'ouvrage est généralement excellente. Les auteurs ont su éviter le style lourd et empesé qu'on retrouve trop souvent dans les livres à caractère juridique. On y retrouve bien sûr quelques expressions particulièrement ésotériques, comme «l'inéluctable Janus», mais qui ne limitent en rien l'accessibilité de l'ouvrage. Celui-ci est particulièrement volumineux, dépassant largement le millier de pages. De plus, il est rempli de renvois référant systématiquement le lecteur aux différents endroits du volume où est abordé un même sujet. Pour limiter les inconvénients inhérents à la consultation d'un ouvrage aussi volumineux, le livre est accompagné d'une table des matières bien conçue et parfaitement accessible figurant en début de volume. De plus, chaque titre est lui-même précédé d'une table des matières. Par ailleurs, le livre est accompagné de trois tables aussi exhaustives qu'utiles : une table des principales lois citées, une table alphabétique et une table de la jurisprudence citée.

Un livre d'une telle ampleur se périme malheureusement rapidement. La branche de droit dont il traite est l'objet de fréquents changements tant d'origine législative que prétorienne. C'est pourquoi il faut inciter les auteurs à ne pas tarder à élaborer une deuxième édition. C'est l'inéluctable contrepartie à la qualité et à l'utilité de l'ouvrage qu'ils ont mis à la disposition des lecteurs intéressés d'une façon ou d'une autre au droit de l'emploi du Québec.

Gilles Trudeau

Université de Montréal 